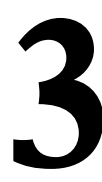

\title{
LA NATURALEZA DE LA CIENCIA Y LOS LIBROS DE TEXTO DE CIENCIAS: UNA REVISIÓN
}

\author{
(NATURE OF SCIENCE AND SCIENCE TEXTBOOKS: A REVIEW \\ OF RESEARCH)
}

Joan Josep Solaz-Portolés

IES Benaguasil, Valencia

\section{RESUMEN}

Este trabajo se inicia con la importancia del libro de texto en los procesos de enseñanza/aprendizaje de las ciencias y la relevancia que tiene la comprensión de la naturaleza de la ciencia en dichos procesos. A continuación, se muestran las ideas sobre epistemología e historia de la ciencia que los expertos consideran imprescindibles en un currículum de ciencias. Seguidamente, se recopilan los estudios que han analizado la naturaleza de la ciencia que presentan los libros de texto. Los textos tienen muchas deficiencias en este aspecto, por lo que se propone actuar en la formación del profesorado.

\section{ABSTRACT}

This paper begins stating the importance of textbook in the processes of science teaching/learning and the relevance of understanding the nature of science in these processes. The article also emphasises the ideas on epistemology and history of science that are considered essential in a science curriculum by experts. Finally, the studies that have analysed how nature of science is presented in text books are compiled. The textbooks have many deficiencies in this aspect, and because of this reason, measures regarding the field of teachers training are proposed. 


\section{INTRODUCCIÓN}

Es bien conocido que el libro de texto constituye un material curricular de gran relevancia en el aula de ciencias. Así, por ejemplo, en la educación secundaria parece ser el material curricular de mayor influencia (Perales, 2006). Una muestra de la importancia que se le da al libro de texto en la enseñanza de las ciencias es que él mismo se ha convertido en objeto de estudio para los investigadores en la didáctica de las ciencias. Así, por ejemplo, pueden encontrarse trabajos en los que se analizan las características que mejoran su comprensión y aprendizaje (Solaz-Portolés, 2009), se evalúan los contenidos de acuerdo con las líneas que plantean las reformas educativas (Vázquez, Ruipérez y Nuño, 1998), o se discuten cómo se introducen los conceptos científicos (Solaz-Portolés, 2007).

El célebre filósofo e historiador de la ciencia T.S. Kuhn (1987a) defiende la tesis de que la enseñanza basada en el libro de texto permite la formación de científicos aptos para realizar ciencia normal y los adiestra como solucionadores de problemas. Este autor, llega a definir a los libros de texto como "vehículos pedagógicos para la perpetuación de la ciencia normal" (Khun, 1987a, 214). En otro de sus trabajos, lo define como "la única fuente mediante la cual la mayoría de las personas entran en contacto con las ciencias físicas (...). Como atestiguan muchas autobiografías, incluso el investigador científico no siempre se halla libre de la imagen de libro de texto obtenida durante sus primeros contactos con la ciencia“ (Kuhn, 1987b, 204).

No obstante, los estudiantes suelen tener muchas dificultades para comprender los textos de ciencias (Snow, 2002). Esto no ha de resultar extraño atendiendo a los resultados obtenidos en un estudio (Roseman, Kevidou, Stern y Cadwell, 1999) auspiciado por la American Association for Advancement of Science (AAAS). En dicho estudio, se evaluó la calidad instruccional de libros de texto de grado medio utilizados en USA. Los resultados no pudieron ser más desoladores: la gran mayoría no satisfacía los mínimos exigibles.

Por otra parte, en opinión de Martín-Díaz (2002) para una adecuada formación científica uno de los requisitos necesarios es ofrecer a los estudiantes una apropiada concepción de la ciencia. Para la autora, se tendría que incidir especialmente en los siguientes aspectos de la construcción del conocimiento científico: intenta representar la realidad, es una práctica social no ajena a otras actividades sociales, y se ha de cuestionar su objetividad y neutralidad. En esta misma línea, Alan Leshner, Director de la AAAS, afirma en una entrevista que en la educación secundaria comprender la naturaleza de la ciencia es incluso más importante que dominar sus detalles 
(Perkins-Gough, 2007). La OCDE reconoce la importancia de comprender los procesos de la ciencia que generan conocimiento y proponen explicaciones sobre el mundo natural (OECD, 2006, 21).

Además, las investigaciones llevadas a cabo por Driver, Leach, Millar y Scott (1996), y por McComas, Almazroa y Clough (1998), muestran que las explicaciones sobre la naturaleza de la ciencia mejoran el aprendizaje de los contenidos científicos. Solbes y Traver (2001) concluyen que la introducción de la historia de la ciencia en el aula mejora la imagen de la ciencia y genera actitudes positivas hacia ella.

En este trabajo, nos proponemos, en primer lugar, destacar las ideas más importantes sobre epistemología e historia de la ciencia que, de acuerdo con los expertos, se deberían incluir en un currículum de ciencias. En segundo lugar, analizaremos los trabajos aparecidos en distintas revistas de educación científica en los que se investigan los contenidos de los libros de texto de distintos niveles educativos y su relación con las características del conocimiento científico y su desarrollo histórico. Finalmente, extraeremos las oportunas conclusiones y las consecuencias que se deriven para la enseñanza.

\section{EPISTEMOLOGÍA E HISTORIA DE LA CIENCIA EN EL CURRÍCULUM DE CIENCIAS: OPINIÓN DE LOS EXPERTOS}

En el estudio de Osborne, Collins, Ratcliffe, Millar y Duschl (2003) se determina el grado de acuerdo entre profesores de ciencias y de educación científica, filósofos y sociólogos de la ciencia a la hora de decidir qué contenidos sobre las características del conocimiento científico, metodología de la ciencia e instituciones y prácticas sociales han de ser incluidos en el currículo de ciencias. El estudio recomienda las ideas que deben ser recogidas en el currículo, que son:

- Ciencia y certeza.

- Desarrollo histórico del conocimiento científico.

- Base empírica de la ciencia.

- Metodología científica y contrastación crítica.

- Diversidad de pensamiento científico.

- Análisis e interpretación de datos.

- Hipótesis y predicción.

- Creatividad. 
- Ciencia y pensamiento crítico.

- Cooperación y colaboración en el desarrollo del conocimiento científico.

Por su parte Lederman (2004) separa, por una parte, la naturaleza de la ciencia, y por otra, la indagación científica. Con todo, admite que ambas están íntimamente relacionadas y se solapan. Este autor usa tres criterios para determinar los puntos de la naturaleza de la ciencia a incluir en el currículo: ¿pueden ser comprendidos por los estudiantes?, ¿hay un consenso general sobre ellos? y, ¿son útiles para los ciudadanos? Los puntos que destaca este investigador son:

- El conocimiento científico es tentativo.

- La ciencia está embebida del contexto social y cultural.

- Leyes y teorías científicas.

- Observaciones e inferencias.

- La ciencia está basada en la experiencia.

- La ciencia es subjetiva y cargada de teoría.

- La ciencia implica necesariamente inferencia, imaginación y creatividad.

- La investigación puede ser descriptiva, correlacional y experimental.

- La ciencia utiliza múltiples enfoques y análisis.

Por último, en el trabajo de Acevedo-Díaz, Vázquez, Manassero y Acevedo-Romero (2007) se presentan los acuerdos conseguidos en una investigación realizada con expertos españoles sobre diferentes cuestiones acerca de la naturaleza de la ciencia. La búsqueda se ha centrado en los aspectos de la naturaleza de la ciencia que se estiman adecuados, así como en los que se consideran mitos, visiones deformadas o creencias inadecuadas. Destacamos los siguientes aspectos en los que se logra el consenso:

- Las observaciones dependen de la teoría.

- Los modelos científicos no son la realidad.

- El conocimiento científico es dinámico y provisional.

- Las teorías científicas no tienen por qué ser ni simples ni elegantes.

- La ciencia progresa tanto de ideas que se confirman como de ideas que se refutan. 
- La originalidad y creatividad forman parte del desarrollo del trabajo científico.

De acuerdo con Monk y Osborne (1997), los materiales curriculares que presentan el desarrollo de la ciencia en su contexto histórico y sociocultural tienen un gran potencial para mejorar el aprendizaje de conceptos científicos y de la naturaleza del conocimiento científico. Fernández (2000) resume las conclusiones de los distintos investigadores en relación a la utilización de la historia de la ciencia en la enseñanza:

- Fomenta actitudes positivas hacia la ciencia.

- Facilita la comprensión de la ciencia.

- Pone de relieve la dimensión humana de la ciencia.

- Atenúa el dogmatismo.

- Posibilita la asimilación de la naturaleza, métodos y evolución de la ciencia.

- Permite anticipar dificultades y concepciones de los estudiantes.

- Sugiere propuestas didácticas.

- Orienta en la selección, secuenciación y exposición de los contenidos.

Un aspecto de la historia de la ciencia que merece especial atención en el currículo de ciencias es la participación de las mujeres en la ciencia (Pérez, 1992). Se hace necesaria la recuperación de figuras silenciadas y olvidadas, y la reflexión sobre la exclusión de las mujeres en la ciencia y la tecnología, a pesar de sus contribuciones notables a ciertos problemas científicos (González y Pérez, 2002).

\section{ESTUDIOS SOBRE LA NATURALEZA DE LA CIENCIA EN LOS LIBROS DE TEXTO DE CIENCIAS}

Hemos revisado los trabajos aparecidos en las revistas de educación y educación científica en los que se tratan cuestiones epistemológicas y/o históricas de la ciencia en los libros de texto de distintos países y niveles académicos. Hemos tomado como punto de partida la década de los ochenta (1988), y hemos llegado hasta el año actual, 2009. Seguiremos un orden cronológico en la revisión de los estudios.

Meheut, Larcher y Chomat (1988) investigaron la presentación de los modelos científicos en los libros de texto franceses y escoceses. En los ma- 
nuales franceses los modelos científicos son presentados como objetos reales, ocultando las reglas de correspondencia que fundamentan su validez y sus aspectos teóricos. En un gran número de manuales escoceses se adopta un punto de vista empirista, y se espera que a partir de determinadas experiencias el alumno llegue a construir por sí mismo un modelo.

Strube (1989) efectuó un análisis de argumentos y explicaciones presentados en textos de Física y Química (preuniversitarios y universitarios) ingleses y australianos, y en él se señala la ausencia de pasajes que discutan la función de teorías, leyes y modelos, en los procesos de indagación científica. Además, revela que la mayoría de los textos contienen un alto índice de explicaciones no justificadas (unjustified statements) que son utilizadas para informar al lector de resultados, y un bajo índice de explicaciones indagativas (inquiry statements), donde se describen las dificultades conceptuales y experimentales que acompañaron el desarrollo de los conceptos científicos.

Chiappetta, Sethna y Fillman (1991) tras el examen del contenido de siete textos de Química de High School (USA) para determinar la inclusión de cuatro aspectos de la naturaleza de la ciencia (ciencia como un cuerpo de conocimientos, ciencia como un modo de investigación, ciencia como un modo de pensamiento, y ciencia y sus interacciones con la sociedad y la tecnología), concluyen que: hacen mucho énfasis en el contenido científico y poco en los aspectos relacionados con la investigación; presentan un cuerpo de conocimientos elaborados sin ningún tipo de error, es decir, ofrecen una visión acumulativa de la ciencia; y no prestan importancia alguna al desarrollo histórico de los conceptos.

Stinner (1992) revisó libros de texto de ciencias de Canadá, y observó que ofrecen una falsa impresión de la naturaleza de la ciencia: presentan un cuerpo de conocimiento elaborado de forma empírico-inductiva y acompañado de resolución de problemas de carácter algorítmico mayoritariamente.

Solaz-Portolés, Sanjosé y Vidal-Abarca (1993) analizaron en textos de Física y Química de educación secundaria españoles la introducción de los modelos atómicos, y encontraron que no se dan a conocer los aspectos epistemológicos de la formulación de modelos científicos, ni se justifica la sustitución de un modelo por otro atendiendo a las dificultades que uno presentó y el otro obvió.

Chiappetta, Sethna y Fillman (1993) volvieron a buscar los cuatro aspectos de la naturaleza que ya aplicaron en un trabajo ya citado (Chiappetta, Sethna y Fillman,1991), esta vez en textos de ciencias de la Middle School (USA). Obtienen que la mayoría de textos trata muy poco las interacciones 
Ciencia/Técnica/Sociedad y una visión de la metodología científica en pasos estereotipados.

Whiteley (1996a, 1996b), realizó sendos trabajos sobre la presencia de la mujer en los libros de texto de ciencias de la educación secundaria. En el primero de ellos (Whiteley, 1996a), detecta la escasísima presencia de científicas en los libros de texto de Física en el Caribe y en el Reino Unido. En el segundo, obtiene los mismos resultados (Whiteley, 1996b) en los textos de ciencias de los High Schools Jamaicanos.

Cutrera y Dell'Oro (2001) buscaron en los libros de texto argentinos de ciencias de distintos niveles educativos la presencia del contexto de descubrimiento científico en sus páginas. La mayoría de los textos no ofrecen cuestiones relacionadas con el contexto de descubrimiento, y en aquellos que lo hacen, prevalece una visión inductivista ingenua. Una parte importante de los textos no considera la creatividad como fundamental en la generación del conocimiento científico y simplifica en extremo la metodología científica, orientándola hacia el inductivismo ingenuo.

Knain (2001) indagó en textos de ciencias noruegos de octavo grado qué imagen de la ciencia proporcionaban. Encontró que la mayoría de los textos potencia una imagen individualista, donde el científico realiza experimentos que le conducen a importantes descubrimientos. Se suele ignorar los aspectos sociales de la ciencia. Solamente un texto presenta al conocimiento científico fruto del consenso de la comunidad científica e indica las influencias sobre éste de la economía, la sociedad, y los valores personales.

Cutrera (2003) analizó la visión de la validación del conocimiento científico presentada en los libros de texto escolares argentinos. Mayoritariamente se observa que la validación del conocimiento científico se hace recaer sobre la experiencia. Son muy pocas las referencias al papel de la comunidad científica en la aceptación y validación del conocimiento científico.

Cutrera y Dell'Oro (2003) investigaron la información ofrecida en los textos escolares argentinos de ciencias sobre el método científico. Sus resultados ponen de relieve que se privilegia una visión del método científico entendido como un procedimiento algorítmico destinado a generar y validar el conocimiento científico. De este modo, se genera una imagen estereotipada y errónea de la actividad científica.

McComas (2003) buscó y analizó en los libros de textos de Biología de educación secundaria estadounidenses las definiciones de ley y teoría, que son de vital importancia en la ciencia. Para ello, revisó en la literatura de filosofía de la ciencia tales definiciones y las comparó con las que aparecían 
en los libros. Obtuvo que el término ley raramente aparece definido en los textos, y las definiciones de teoría, que sí suelen darse, son muy diversas aunque ningún texto menciona el aspecto creativo o de invención que hay en la construcción de las teorías.

Rodríguez y Níaz (2004) evaluaron la presentación de los modelos atómicos en libros de texto de Física publicados en USA, basándose en criterios derivados de la historia y filosofía de la ciencia. Un elevado porcentaje de textos ofrece una perspectiva inductivista en la que lo más destacado son los detalles experimentales. Muy pocos textos contienen una reconstrucción histórica que incluya tanto el contexto en el que se llevaron a cabo los experimentos, como los fundamentos teóricos que guiaron a los científicos y las interpretaciones alternativas de los datos experimentales que pueden conducir a conflictos o controversias.

Malaver, Pujol y D'Alessandro (2004) estudiaron, entre otras cosas, la imagen de la ciencia en textos de Química de los cursos básicos universitarios en Venezuela. Se concluye en su trabajo que el contenido científico en dichos textos se presenta en términos de conceptos, principios, leyes, y teorías, sin que se tome en consideración los problemas históricos y/o epistemológicos que originaron el desarrollo del conocimiento científico, ni las hipótesis que se plantearon en su elaboración.

Guisasola y Furió (2005) examinaron cómo se introducen los conceptos y teorías del campo magnético en los libros de texto de Física de los primeros cursos universitarios utilizados en España. Sus resultados muestran que no se hace una introducción acorde con la naturaleza de la ciencia, y señalan cómo el desarrollo de la teoría del campo magnético puede servir, precisamente, para ilustrar las características de la naturaleza de la ciencia.

Chiappeta y Fillman (2006) vuelven, nuevamente, a utilizar los cuatro aspectos de la naturaleza de la ciencia que ya aplicaron en anteriores trabajos (Chiappetta, Sethna y Fillman 1991, 1993), en esta ocasión sobre textos de Biología de High School (USA) de reciente publicación. Además, comparan los resultados con los obtenidos con textos de la misma asignatura publicados quince años atrás. Destacan una mejora considerable de los contenidos relacionados con la naturaleza de ciencia y la adecuación de éstos a las guías para la reforma de la educación científica en USA.

Abd-El-Khalick, Waters y Le (2008) llevaron a cabo un estudio en el que se evaluaron libros de texto de Química utilizados durante las cuatro décadas pasadas en los High School de USA. En dicha evaluación se emplearon diez ideas básicas sobre la naturaleza de la ciencia (diez ítems), con una escala cuya amplitud iba de -3 a 3 ( -3 para las ideas totalmente erróneas o 
ingenuas presentes en el texto, y 3 para las ideas completas y correctas). Por tanto, la puntuación final de un texto podía fluctuar entre -30 y 30 . Sus resultados son sorprendentes: ningún texto puntuó más alto de 12 y ese texto fue publicado en 1968. Las ideas básicas sobre la naturaleza de la ciencia son:

- Las observaciones de los científicos están filtradas por la percepción humana, los instrumentos científicos y las bases teóricas que los sustentan.

- No es lo mismo una observación que una inferencia. Las construcciones científicas son inferenciales, esto es, sólo se puede acceder a ellas o ser medidas mediante sus efectos.

- Generar conocimiento científico implica creatividad, como pueden ser propuestas de explicaciones o entidades teoréticas.

- La base teórica, las creencias, el conocimiento previo, la formación y las expectativas tienen un papel decisivo en el trabajo del científico.

- El conocimiento científico es fiable y duradero, pero nunca absoluto ni cierto.

- No existe un método científico que garantice la producción de conocimiento.

- Las teorías científicas han sido contrastadas empíricamente, son consistentes internamente, generan cuestiones y problemas para investigar, y guían futuras investigaciones.

- Las leyes son proposiciones que contienen relaciones entre variables y que forman parte de una teoría.

- El conocimiento científico es fruto del consenso de la comunidad científica, por tanto está socialmente negociado.

- La ciencia es una empresa humana y, como tal, está impregnada del contexto cultural, social, económico y filosófico dominante.

Por último, Irez (2009) investigó en libros de texto de Biología de la educación secundaria de Turquía el tratamiento dado a la naturaleza de la ciencia. Utilizó un análisis etnográfico del contenido y los datos los analizó mediante mapas cognitivos. La investigación revela que la ciencia es generalmente representada como una mera colección de hechos, no como un conjunto de procesos dinámicos de generación y puesta a prueba de explicaciones sobre la naturaleza. 


\section{UN EJEMPLO ILUSTRATIVO: LA METODOLOGÍA CIENTÍFICA EN UN LIBRO DE TEXTO ESPAÑOL DE TERCERO DE LA EDUCACIÓN SECUNDARIA OBLIGATORIA (ESO)}

Presentamos a continuación, a título de ejemplo, un fragmento que consta bajo el epígrafe El trabajo en las ciencias experimentales: el método científico en un texto educativo de Física y Química de $3 .^{\circ}$ de la ESO (Física y Química 3. ${ }^{\circ}$ ESO, Proyecto La casa del Saber, Editorial Santillana, 2008). En él pueden observarse varios aspectos de la visión simplista y deformada de la ciencia que han aparecido anteriormente (carácter algorítmico del quehacer científico, no se destaca que las observaciones de los científicos están basadas en un cuerpo teórico, perspectiva inductivista, etc.).

El método científico sigue una serie de pasos. Los más importantes o generales son los que siguen:

1. Observación. Consiste en examinar y analizar un fenómeno concreto planteándose preguntas sobre éste.

2. Elaboración de hipótesis. Consiste en dar una posible explicación del fenómeno y responder a las cuestiones planteadas. Cada respuesta posible es una hipótesis.

3. Experimentación. Se intentan reproducir los fenómenos observados en condiciones controladas para verificar si las hipótesis son verdaderas. Algunas veces, el Universo es el laboratorio (volcanes, terremotos...).

4. Análisis de los resultados. En la actualidad, los ordenadores permiten efectuar cálculos fácilmente, manejar tablas y representar los datos obtenidos en un experimento.

5. Obtención de conclusiones, definición de leyes y establecimiento de teorías. En muchos casos, estas conclusiones predicen algún fenómeno observable en el futuro. Por ejemplo, Mendeleiev predijo la existencia de elementos nuevos todavía desconocidos en aquella época.

6. Publicación de resultados. El objetivo es que otros científicos puedan reproducir los resultados o utilizarlos para sus estudios. Para que la ciencia avance es imprescindible compartir los conocimientos con los otros. Hoy en día, Internet y las publicaciones científicas hacen posible que cualquier avance significativo sea conocido casi al instante por todos los científicos del mundo. 


\section{CONCLUSIONES Y DISCUSIÓN}

En los trabajos que hemos revisado, hemos podido comprobar que se han analizado en los libros de texto prácticamente todos los aspectos sobre las características del conocimiento científico, metodología de la ciencia, sus interacciones y modo de crecimiento que, en opinión de los expertos, resultan de especial relevancia para la enseñanza/aprendizaje de las ciencias. Como acabamos de ver, las deficiencias de los textos en esta cuestión no conocen fronteras, ni niveles académicos. Solamente podemos exceptuar los textos de Biología de High School (USA) publicados en 2005-2006 (Chiappeta y Fillman, 2006).

Destacamos a continuación las defectos que con mayor frecuencia aparecen en los libros de texto en relación con la naturaleza de la ciencia y que, en consecuencia, deberían ser tenidas en cuenta tanto por los autores de libros de texto, como por el profesorado cuando efectúa (o tendría que efectuar) un análisis crítico del libro de texto ante sus alumnos. Estos defectos son:

- Falta de explicaciones adecuadas de la función de las teorías, leyes y modelos.

- Los modelos científicos aparecen como parte de la realidad física, sin mostrar su base teórica.

- Presentación de la generación del conocimiento científico sin ausencia de errores, de forma empírico-inductiva y sin resaltar que la teoría siempre guía al científico en sus acciones.

- Énfasis en la imagen algorítmica de la metodología científica

- Poca información acerca de la participación de las mujeres en el desarrollo de la ciencia.

- Se destaca poco la creatividad como cualidad en la producción del conocimiento científico.

- No se incide explícitamente en la consideración de la ciencia como conjunto de procesos dinámicos donde se ponen a prueba diversas explicaciones (hipótesis).

- No se muestra la ciencia como una actividad social que crea el conocimiento no sólo a partir de la actividad experimental, sino también del consenso de la comunidad científica.

- No se recogen las influencias de otras disciplinas (economía, filosofía, etc.) y de la sociedad (instituciones políticas, empresas, etc.) sobre la producción científica. 
Podemos, pues, afirmar que, en general, la imagen de la ciencia que los libros de texto proporcionan es distorsionada y fruto del vacío histórico y filosófico que muestran sus páginas. En estas condiciones, y teniendo presente el papel decisivo que ha tenido y tiene el libro de texto en la formación científica, resulta más fácil entender las ideas erróneas acerca de la naturaleza de la ciencia que sostienen en nuestro país los estudiantes (Vázquez y Manassero, 1999); los profesores en formación inicial, tanto los de primaria (Guisasola y Morentín, 2007), como los de secundaria (Acevedo, 2000); y los profesores en ejercicio (Manassero y Vázquez, 2000).

A la vista de la importancia en la enseñanza/aprendizaje de las ciencias de una adecuada comprensión de la naturaleza de la ciencia, y de los constatados déficits de los libros de texto en este punto, parece necesario tomar las medidas pertinentes para mejorar los contenidos de los libros de texto relacionados con la naturaleza de la ciencia. Creemos que para lograr este objetivo se hace imprescindible actuar en la formación inicial y permanente del profesorado, mediante las acciones oportunas que permitan la preparación adecuada de los docentes en epistemología, historia y sociología de la ciencia. Hay que tener presente la escasa o nula presencia de estos aspectos de la ciencia en la formación científica y docente actual y, lo que es peor, no hay indicios que esta situación vaya a mejorar con los nuevas maestrías o máster de formación del profesorado. 


\section{REFERENCIAS BIBLIOGRÁFICAS}

Abd-El-Khalick, F., Waters, M., y Le, A. P. (2008). Representations of nature of science in High School Chemistry textbooks over the past four decades. Journal of Research in Science Teaching, 45 (7), 835-855.

Acevedo, J.A. (2000). Algunas creencias sobre el conocimiento científico de los profesores de Educación Secundaria en formación inicial. Bordón, 52 (1), 5-16.

Acevedo-Díaz, J. A., Vázquez, A., Manassero, M.A., y Acevedo-Romero, P. (2007). Consensos sobre la naturaleza de la ciencia: Aspectos epistemológicos. Revista Eureka sobre Enseñanza y Divulgación de las Ciencias, 4 (2), 202225. Disponible en: http://www.apaceureka.org/revista/Volumen4/ Numero_4_2/Acevedo_et_al_2007.pdf [consulta 2009, 28 de Marzo].

Chiappetta, E. L. y Fillman, D. A. (2007). Analysis of five high school biology textbooks used in the United States for inclusion of the nature of science. International Journal of Science Education, 29 (15), 1847-1868.

Chiappetta, E. L., Sethna, G. H., y Fillman, D. A. (1991). A quantitative analysis of high school chemistry textbooks for scientific literacy themes and expository learning aids. Journal of Research in Science teaching, 28, 939-951.

Chiappetta, E. L., Sethna, G. H., y Fillman, D. A. (1993). Do middle school life science textbooks provide a balance of scientific literacy themes? Journal of Research in Science Teaching, 30, 787-797.

Cutrera, G. (2003). La justificación del conocimiento científico en los textos escolares. Revista Iberoamericana de Educación (en línea). Disponible en: http://www.rieoei.org/experiencias46.ht m [consulta 2009, 7 de Marzo].
Cutrera, G., Dell'Oro, G. (2001). El contexto de descubrimiento en los textos escolares. Revista Iberoamericana de Educación (en línea). Disponible en: http://www.rieoei.org/experiencias17.ht m [consulta 2009, 7 de Marzo].

Cutrera, G., Dell'Oro, G. (2003).Un análisis de contenido en textos escolares sobre el método científico. Revista Iberoamericana de Educación (en línea). Disponible en: http://www.rieoei. org/experiencias55.htm [consulta 2009, 7 de Marzo].

Driver, R., Leach, J., Millar, R., y Scott, P. (1996). Young people's images of science. Buckingham: Open University Press.

Fernández, M. (2000). Fundamentos históricos. En F. J. Perales y P. Cañal (Eds.) Didáctica de las Ciencias Experimentales. Alcoy: Editorial Marfil.

González, M. I. y Pérez, E. (2002). Ciencia, Tecnología y Género. Revista Iberoamericana de Ciencia, Tecnología, Sociedad e Innovación (en línea), 2. Disponible en: http://www.oei.es/ revistactsi/numero2/varios2.htm [consulta 2009, 10 de Marzo].

Guisasola, J. y Furió, C. (2005). The nature of science and its implications for Physics textbooks: The case of classical magnetic field theory. Science \& Education, 14 (3), 321-328.

Guisasola, J. y Morentin, M. (2007). ¿Comprenden la naturaleza de la ciencia los futuros maestros y maestras de Educación Primaria? Revista Electrónica de Enseñanza de las Ciencias (en línea), 6 (2), 246-262. Disponible en: http://www.saum.uvigo.es/reec/volume nes/volumen6/ART2_Vol6_N2.pdf [consulta 2009, 1 de Abril].

Irez, S. (2009). Nature of science as depicted in Turkish Biology textbooks. Science Education, 93 (3), 422-447. 
Knain, E. (2001). Ideologies in school science textbooks. International Journal of Science Education, 23, 319-329.

Kuhn, T. S. (1987a). La estructura de las revoluciones científicas (11. ${ }^{\text {a }}$ Reimpresión). Madrid: FCE.

Kuhn, T. S. (1987b). La tensión esencial (1 ${ }^{a}$ Reimpresión). México, D.F.: FCE.

Lederman, N. G. (2004). Syntax of nature of science within inquiry and science instruction. En L. B. Flick y N. G. Lederman (Eds.), Scientific Inquiry and Nature of Science. Bordrecht: Kluwer Academic Publishers, 301-317.

Malaver, M., Pujol, R., y D'Alessandro, A. (2004). Imagen de la ciencia y vinculaciones Ciencia-Tecnología-Sociedad en textos universitarios de Química General. Revista de Pedagogía (en línea), 25, Disponible en: http://www. scielo.org.ve/scielo.php?script $=$ sci arttext\&pid $=$ S0798-97922004000 $100004 \& \operatorname{lng}=$ es \&nrm $=$ iso [consulta 2009, 28 de Febrero].

Manassero, M. A. y Vázquez, A. (2000). Creencias del profesorado sobre la naturaleza de la ciencia. Revista Interuniversitaria de Formación del Profesorado, 37, 187-208.

Martín-Díaz , M. J. (2002). Enseñanza de las Ciencias, ¿para qué? Revista Electrónica de Enseñanza de las Ciencias (en línea), 1 (2). Disponible en: http://www.saum.uvigo.es/reec/volume nes/volumen1/Numero2/Art1.pdf [consulta 2009, 15 de Marzo].

McComas, W. F. (2003). A textbook case of the nature of science: Laws and theories in the science of Biology. International Journal of Science and Mathematics Education, 1 (2), 141-155.

McComas, W. F., Almazroa, H., y Clough, M. (1998). The nature of science in science education: An introduction. Science \& Education, 7 (6), 511-532.

Meheut, M., Larcher, C. y Chomat, A. (1988). Modelos de partículas en la ini- ciación a las ciencias físicas. Enseñanza de las Ciencias, 6, 231-238.

Monk, M., y Osborne, J. (1997). Placing the history and philosophy of science on the curriculum: A model for the development of pedagogy. Science Education, 82, 527-552.

Organisation for Economic Co-operation and Development (OECD), (2006). Assessing Scientific, Reading and Mathematical Literacy: A Framework for PISA 2006. Disponible en: www.oecd.org/ document/33/0,3343, en_32252351_ 32236191_37462369_1_1_1_1,00.html [consulta 2009, 28 de Febrero].

Osborne, J., Collins, S., Ratcliffe, M., Millar, R., y Duschl, R. (2003). What "Ideas about Science" should be taught in school science?. A Delphi study of the expert community. Journal of Research in Science Teaching, 40, 692-720.

Perales, F. J. (2006). Uso (y abuso) de la imagen en la enseñanza de las ciencias. Enseñanza de las Ciencias, 24 (1), 13-30.

Pérez, E. (1992). La enseñanza de la historia de las ciencias y los estudios sobre la mujer. Revista da Sociedade Brasileira de História da Ciencia, 7, 25-30.

Perking-Gough, D. (2007). Understanding the Scientific Enterprise: A Conversation with Alan Leshner. Educational Leadership, 64 (4), 8-15.

Rodríguez, M. A. y Niaz, M. (2004). A reconstruction of structure of the atom and its implications for General Physics textbooks: A history and philosophy of science perpective. Journal of Science Education and Technology, 13 (3), 409-424.

Roseman, J. E., Kevidou, S., Stern, L. \& Caldwell, A. (1999). Heavy books on learning: AAAS Project 2061 Evaluates Middle Grades Science Textbooks. Science Books \& Films (en línea), 35 (6). Disponible en: http://www.project 2061.org/publications/textbook/articles/ heavy.htm [consulta 2009, 10 de Marzo]. 
Snow, C. (2002). Reading for understanding: Toward an R\&D program in reading comprehension. Santa Monica, CA: RAND Corporation.

Solaz-Portolés, J. J. (2007). Algunas deficiencias en el tratamiento del equilibrio químico en los libros de texto preuniversitarios españoles de Química. Revista Chilena de Educación Científica, 6 (1), 13-21.

Solaz-Portolés, J. J. (2009). Aprender ciencia con textos: Bases teóricas y directrices. Latin American Journal of Physics Education, 3 (2), 376-379. Disponible en: http://www.journal.lapen.org.mx [consulta 2009, 25 de Mayo]

Solaz-Portolés, J. J., Vidal-Abarca, E., y Sanjosé, V. (1993). Análisis didáctico, epistemológico e histórico de la introducción de los modelos atómicos en 2. ${ }^{\circ}$ de BUP. Enseñanza de las Ciencias, Número extra (IV Congreso), 129-130.

Solbes, J. y Traver, M. (2001). Resultados obtenidos introduciendo la historia de la ciencia en las clases de Física y Química: Mejora de la imagen de la ciencia y desarrollo de actitudes positivas. Enseñanza de las Ciencias, 19 (1), 151-162.
Stinner, A. (1992). Science textbooks and science teaching: From logic to evidence. Science Education, 73, 591-605.

Strube, P. (1989). A content analysis of arguments and explanations presented to students in physical science textbooks: A model and a example. International Journal of Science Education, 11, 195-202.

Vázquez, J. R., Manassero, M. A. (1999). Características del conocimiento científico: Creencias de los estudiantes. Enseñanza de las Ciencias, 17 (3), 377395.

Vázquez, J. R., Ruipérez, T. y Nuño, M. T. (1998). La reforma en los libros de texto de Ciencias Naturales de la ESO. Revista de Psicodidáctica, 5, 115-124.

Whiteley, P. (1996a). The gender balance of physics textbooks: Caribbean and British books, 1985-91. Physics Education, 31, 169-174.

Whiteley, P. (1996b). The gender fairness of integrated science textbooks used in Jamaican High Schools. International Journal of Science Education, 18 (8), 969-976. 


\section{PALABRAS CLAVE}

Libros de texto de ciencias, naturaleza de la ciencia, enseñanza/aprendizaje de la ciencia, análisis de libros de texto.

\section{KEYWORDS}

Science textbooks, nature of science, science teaching/learning, textbooks analysis.

\section{PERFIL ACADÉMICO Y PROFESIONAL DEL AUTOR}

Joan Josep Solaz-Portolés es Doctor en Ciencias Químicas (Programa Didáctica de las Ciencias Experimentales) por la Universitat de ValènciaEstudi General. Catedrático de Física y Química en la Educación Secundaria y Profesor-Tutor de la UNED. Ha publicado más de cuarenta trabajos en revistas sobre ciencia, educación y educación científica de once países (algunos de ellas indexadas en SSCI y SCI, la mayoría indexadas en Latindex). Sus actuales líneas de investigación son: Aprendizaje a partir de Textos y Resolución de Problemas. En dichas líneas colabora con el Profesor Dr. Vicente Sanjosé, del Departament de Didáctica de les Ciències de la Universitat de Valencia.

Dirección del autor: C/ Sagunt, 5

46183 L’Eliana (Valencia)

e.mail: jjsolaz@valencia.uned.es

Fecha de Recepción del artículo: 04. mayo. 2009

Fecha de Revisión del artículo: 10. diciembre. 2009

Fecha de Aceptación definitiva del artículo: 15. diciembre. 2009 